\title{
Childhood Immunization Schedule for National Immunization Program Vaccines - China (Version 2021)
}

\author{
National Health Commission of the People's Republic of China
}

\section{GENERAL PRINCIPLES}

\section{Vaccination Age}

The age of administration in the immunization schedule refers to the minimum age for giving a vaccine dose. Vaccines should be administered at the recommended age or as soon as possible after the recommended age. Specific timings and ages for administering certain vaccine doses are shown below and detailed in the second section, "Instructions for Use of Vaccines."

1. The first dose of hepatitis $B(\mathrm{HepB})$ vaccine is to be given within 24 hours of birth.

2. Bacille Calmette-Guérin (BCG) vaccine is given $<3$ months of age.

3. The third dose of HepB vaccine, the third dose of poliovirus vaccine, the third dose of Diphtheria, tetanus, and pertussis (DTaP), the first dose of Measles, mumps, and rubella (MMR), and the first dose of Japanese encephalitis vaccine, live (JE-L) or the second dose of Japanese encephalitis vaccine, inactivated (JE-I) are all to be administered before 12 months of age (during infancy, before the first birthday).

4. The second dose of Group A meningococcal polysaccharide vaccine (MPSV-A) is given $<18$ months.

5. The second MMR dose, the first hepatitis A vaccine, live (HepA-L) or hepatitis A vaccine, inactivated (HepA-I) dose, and the fourth DTaP are all to be given $<24$ months of age (during the second year of life).

6. The second dose of HepA-I, and the second dose of JE-L or the third dose of JE-I are to be given $<3$ years of age (before the third birthday).

7. The first dose of Group A and Group C meningococcal polysaccharide vaccine (MPSV-AC) is to be given $<4$ years of age.

8. The fourth dose of poliovirus vaccine is to be given $<5$ years of age.
9. Diphtheria-Tetanus vaccine (DT), the second dose of MPSV-AC, and the fourth dose of JE-I are to be given $<7$ years of age.

Children who are not vaccinated on time according to the routine schedule shown above should be vaccinated in accordance with the catch-up vaccination principles, described below and given in detail in the second section, "Instructions for Use of Vaccines."

\section{Vaccination Routes}

Vaccines are given in one of several routes that are specific to each vaccine - oral, intramuscular injection (IM), subcutaneous injection (SubQ), or intradermal injection (ID). Refer to "Instructions for Use of Vaccines" for details. The injection sites are normally the deltoid muscle in the lateral aspect of the upper arm or the vastus lateralis muscle in the anterior and lateral aspect of the thigh. When multiple vaccines are given by injection at the same visit (IM, SubQ, or ID), injections may be on the left and right upper arms and the left and right thighs, sequentially. BCG should only be given via the ID route in the upper arm.

\section{Simultaneous Vaccination and Minimum Intervals}

1. When giving more than one injectable vaccine simultaneously, injections should be at different sites. Mixing vaccines in the same syringe prior to injection is strictly forbidden.

2. All National Immunization Program (NIP) vaccines may be given simultaneously according to the immunization schedule and catch-up vaccination principles.

3. It is important to pay attention to the interval between administration of different vaccines. For two or more live vaccines that are not given simultaneously by injection, a minimum interval of 28 days between injections is required. There is no minimum interval between inactivated vaccines or orally administered attenuated live vaccines that are not given simultaneously with other types of inactivated, and 


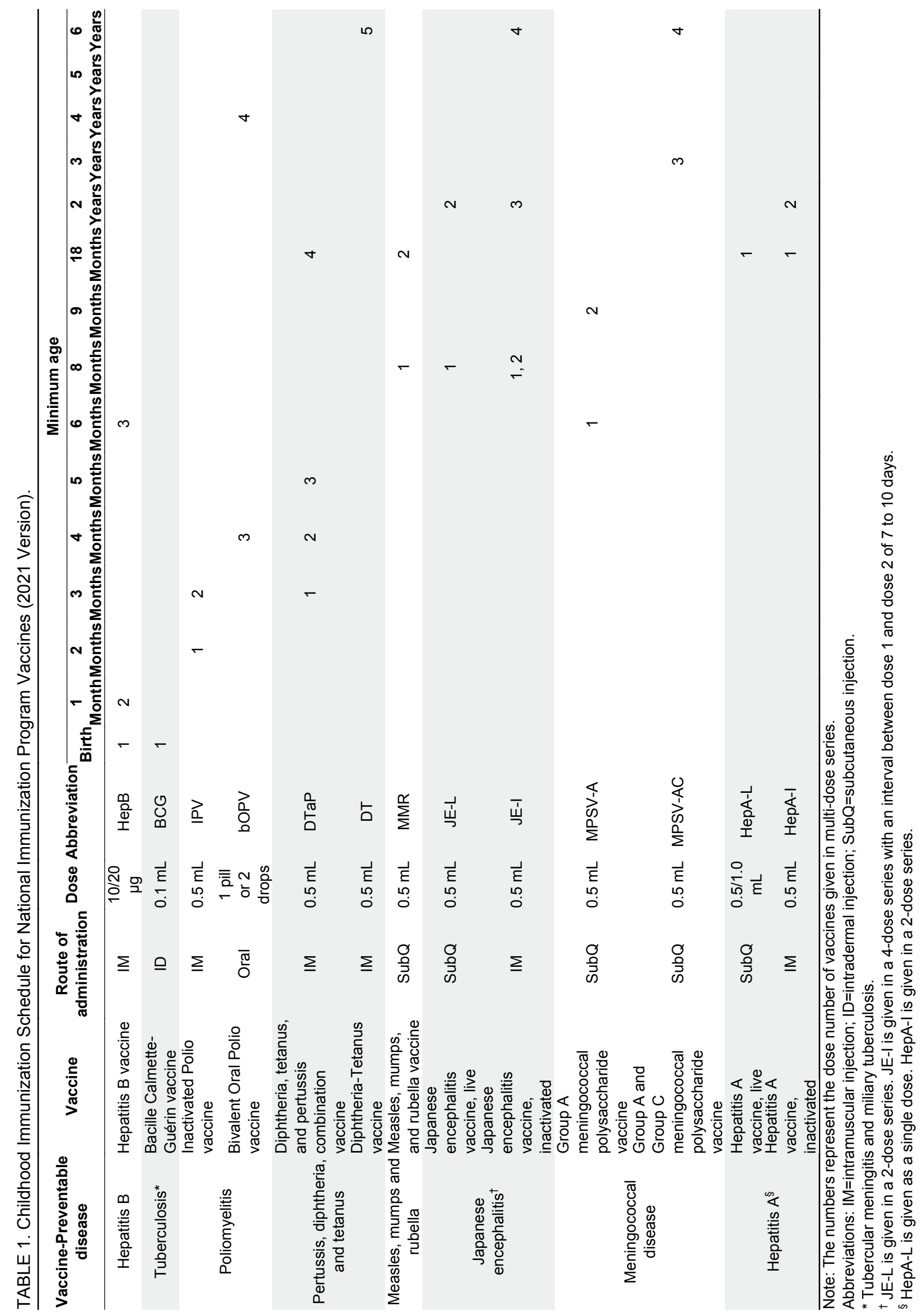


attenuated vaccines (injected or oral administration).

\section{Catch-up Vaccination Principles}

Children below 18 years of age who have not completed their NIP vaccination series by the recommended ages should be caught up based on the following principles.

1. Catch-up vaccination should be accomplished as early as possible to enable children not fully vaccinated to complete the entire vaccine series and be protected at the youngest age possible.

2. It is never necessary to restart a vaccination series; only the remaining doses need to be given.

3. If, when completing a vaccination series, the same vaccine from the same manufacturer is unavailable, the same type of vaccine from a different manufacturer may be used.

4. Additional details are in the next section, "Instructions for Use of Vaccines."

\section{Vaccination During Epidemic Peak Seasons}

NIP vaccines may be given routinely throughout the year, including during the epidemic season, according to the routine immunization schedule, catch-up vaccination principles, requirements of the immunization program, and as required by outbreak response immunization plans.

\section{INSTRUCTIONS FOR USE OF VACCINES}

\section{Recombinant Hepatitis B Vaccine}

\section{Schedule}

HepB vaccine is given in a 3 -dose series at $0,1,6$ months, with the first dose administered within the first 24 hours following birth, the second dose given at 1 month of age, and the third dose given at 6 months of age. HepB is administered by IM injection.

The dose to be administered varies by vaccine type. Recombinant (yeast) HepB is given only in $10 \mu \mathrm{g}$ doses. Newborn infants are given $10 \mu \mathrm{g}$ of $\mathrm{HepB}$ in a single injection, regardless of their mother's hepatitis $\mathrm{B}$ surface antigen ( $\mathrm{HBsAg}$ ) status (positive or negative). Recombinant (CHO cells) HepB is given in two different dose amounts, $10 \mu \mathrm{g}$ or $20 \mu \mathrm{g}$. Babies born to $\mathrm{HBs}$ Ag-negative mothers are given $10 \mu \mathrm{g}$ of $\mathrm{CHO}$ $\mathrm{HepB}$ in a single injection, and babies born to $\mathrm{HBsAg-}$ positive mothers are given $20 \mu \mathrm{g}$ of $\mathrm{CHO} \mathrm{HepB}$ in a single injection.

\section{Notes}

Babies delivered in hospitals should be given the first dose of HepB in the hospital, with the birthing hospital responsible for ensuring vaccine administration. Subsequent doses of HepB are given by the responsible vaccination service center. Newborns not delivered in hospitals are to be given all three HepB doses by the district vaccination service center.

If the mother is $\mathrm{HBsAg}$-positive, 100 units of Hepatitis B hyper-immune globulin (HBIG) are to be administered via intramuscular injection as prescribed by the doctor. HBIG is given with the first dose of HepB vaccine, injected simultaneously in a different limb. HepB, HBIG, and BCG vaccine may be administered simultaneously (at different injection sites).

If the mother is $\mathrm{HBs} A g$-positive or has unknown $\mathrm{HBsAg}$ status, the first dose of HepB should be given as early as possible, within 12 hours of birth. Infants whose birth weight is $<2,000$ grams should receive the first dose of HepB as soon as possible after birth; they should be given an additional 3 doses of HepB ( 4 doses total) beginning at 1 month of age, with the third and fourth doses given at 2 months and 7 months of age.

Newborn infants who are critically ill, have very low birth weights of less than 1,500 grams, have severe birth defects, suffer from severe asphyxia, or have respiratory distress syndrome should be given the first dose of HepB as soon as possible, once vital signs have stabilized.

For infants born to $\mathrm{HBsAg}$ positive mothers who receive post-vaccination serologic testing (PVST) for HBsAg and anti-HBs 1-2 months after the last dose of $\mathrm{HepB}$, if the HBsAg test is negative, and the anti-HBs test is negative or less than $10 \mathrm{mIU} / \mathrm{mL}, 3$ additional doses of HepB can be given at no charge to the family, in accordance with the catch-up schedule.

\section{Catch-up vaccination}

Infants not vaccinated within 24 hours of birth should be vaccinated as soon as possible. Infants who have not received their vaccinations on time should complete the remaining doses as soon as feasible, paying attention to minimum intervals. The minimum interval between dose 1 and dose 2 is 28 days; between dose 2 and dose 3, 60 days; between dose 1 and dose 3 is 4 months.

\section{BCG Vaccine}

1. Schedule

BCG vaccine is recommended for 1 dose, given at 
birth through ID. The dose is $0.1 \mathrm{~mL}$.

2. Notes

Subcutaneous or intramuscular injection is strictly prohibited.

Preterm infants born at gestational age $>31$ weeks and who are in stable condition according to medical evaluation may receive BCG vaccine. Preterm infants born at gestational age $\leq 31$ weeks may be vaccinated before hospital discharge when their condition is stable according to medical evaluation.

There are no special restrictions on the interval between immunoglobulin administration and BCG vaccination.

3. Catch-up vaccination

Infants below 3 months of age may be vaccinated with BCG vaccine.

Children 3 months to 3 years of age whose tuberculin pure protein derivative (TB-PPD)- or Bacilli Calmette Guérin pure protein derivative (BCGPPD) test is negative and who have not been vaccinated should be provided with catch-up vaccination.

Children 4 years of age and older cannot receive BCG catch-up vaccination.

Children who have been vaccinated with BCG cannot receive BCG catch-up vaccination, even in the absence of a BCG scar.

\section{Poliovirus Vaccines (IPV and bOPV)}

\section{Schedule}

Poliovirus vaccination is administered in a 4-dose series - inactivated poliovirus vaccine (IPV) is given at 2 months and 3 months of age followed by bivalent oral poliovirus vaccine (bOPV) given at 4 months and 4 years of age (a 4-dose IPV-IPV-bOPV-bOPV schedule).

IPV is given by IM injection with a dose amount of $0.5 \mathrm{~mL}$; bOPV is given orally as 1 dragée candy or 2 drops of liquid bOPV (approximately $0.1 \mathrm{~mL}$ ).

\section{Notes}

A child who receives a 4-dose-series of IPV or a 4dose-series of an IPV-containing combination vaccine given in a schedule consistent with the vaccine package insert is considered fully vaccinated against poliovirus. It is not necessary to give bOPV to children at 4 years of age if they have completed a 4-dose series of IPVcontaining vaccine.

Some children are recommended to receive only IPV. These children include those with a primary immunodeficiency, who have thymic disease, are living with HIV, have malignant tumors and are undergoing chemotherapy, are currently undergoing hematopoietic stem cell transplantation, are using immunosuppressive or immunomodulating drugs (e.g., high-dose systemic corticosteroids, alkylating agents, anti-metabolic drugs, TNF- $\alpha$ inhibitors, IL-1 blockers, or other immune cell-targeted monoclonal antibodies), or are receiving or have received immune cell-targeted radiation therapy.

\section{Catch-up vaccination}

Children less than 4 years of age who have not received 3 doses of poliovirus vaccine (including catchup vaccination) should complete the 3 -dose series. Children 4 years and older who have not received 4 doses of poliovirus vaccine (including catch-up vaccination) should complete the 4-dose series. For catch-up vaccination, IPV takes precedence over bOPV. The minimum interval between any 2 doses is 28 days. Children who have received 4 doses of polio vaccine after catch-up vaccination should be considered to have completed their full course of polio vaccination.

Children with delayed or missed polio vaccinations but with a vaccination history of trivalent OPV $(\mathrm{tOPV})$, regardless of the number of doses, may be vaccinated with bOPV instead of IPV. For children with no tOPV vaccination history, if they were born before October 1, 2019 (a date when the 2-dose IPV immunization schedule was implemented, and which may vary by PLAD), they should be given 1 dose of IPV; if they were born after October 1, 2019, they should be given 2 doses of IPV.

\section{Schedule}

\section{DTaP and DT Vaccines}

$\mathrm{DTaP}$ and DT are given in a 5-dose series: DTaP at 3 months, 4 months, 5 months, and 18 months of age, and DT at 6 years of age. DTaP and DT are given by IM injection with doses of $0.5 \mathrm{~mL}$.

\section{Notes}

Children who have been vaccinated with a DTaPcontaining combination vaccine according to the vaccine package insert may be considered as to have completed the corresponding dose of $\mathrm{DTaP}$ vaccines.

The vaccine type (DTaP or DT) should be selected based on the age of administration: DTaP is for children aged 3 months to 5 years; child-type DT is appropriate for children aged 6 to 11 years old.

\section{Catch-up vaccination}

Children who did not receive the recommended number of DTaP vaccine doses and who are 3 months 
to 5 years of age should receive the remaining DTaP doses through catch-up vaccination. The minimum interval between any of the first 3 doses is 28 days; the minimum interval between the third and fourth doses is 6 months.

Catch-up vaccination of children 6 years and older is subject to the following principles. Children who received fewer than 3 doses of $\mathrm{DTaP} / \mathrm{DT}$ should receive a cumulative total of 3 doses of DT. Acceptable intervals are 1 to 2 months between the first and second doses, and 6 to 12 months between the second and third doses.

Children who have received 3 or more doses of DTaP/DT cumulatively should be caught up with the following principles. If the child has received at least 1 dose of DT, there is no need for catch-up vaccination. If the child has received only 3 doses of DTaP, he or she should receive 1 dose of DT, with a minimum interval of 6 months between the third dose of DTaP and the DT dose. If the child has received 4 doses of DTaP but no DT vaccine by 7 years of age, he or she should be given 1 dose of DT, with a minimum interval of 12 months between the fourth dose of DTaP and the DT dose.

\section{MMR Vaccine}

\section{Schedule}

Children should receive 2 doses of MMR vaccine the first dose at 8 months and a second dose at 18 months. MMR vaccine is given by subcutaneous injection (SubQ) with a dose amount of $0.5 \mathrm{~mL}$.

\section{Notes}

If a child is in need of MMR and other vaccines, but it is not possible to give all of the vaccines at the same visit, MMR should be the priority vaccine to be administered, and the other vaccines should be given later.

There is a minimum interval of 3 months between administration of immunoglobulin and subsequent administration of MMR vaccine. No immunoglobulin should be given during the 2 weeks after MMR vaccination.

If emergency measles vaccination is necessary according to an epidemiological situation such as an outbreak of measles, children 6 to 7 months of age at risk of measles may receive a single dose of MMR vaccine. This dose is considered a supplemental dose and is not counted as part of routine immunization.

3. Catch-up vaccination

From June 1, 2020, children who have not received
2 doses of MMR vaccine and were born on or after October 1, 2019 should be given MMR to complete the vaccination series.

Children born between 2007 (when MMR vaccine was included in the Expanded Program on Immunization [EPI]) and September 30, 2019, should receive at least 2 doses of measles-containing vaccine, 1 dose of rubella-containing vaccine, and 1 dose of mumps-containing vaccine. MMR should be used to complete the vaccination series if any of the above doses have not been fully received.

Children born before the 2007 inclusion of MMR into EPI, who are under 18 years of age, and have not received 2 doses of measles-containing vaccine, should be given MMR to complete their measles-containing vaccination series.

The minimum interval between MMR doses is 28 days.

\section{JE-L Vaccine}

\section{Schedule}

JE- $\mathrm{L}$ is given as a 2-dose series: the first dose at 8 months and a second dose at 2 years. JE-L is given by subcutaneous injection (SubQ) with a dose amount of $0.5 \mathrm{~mL}$.

\section{Notes}

People living in Qinghai, Xinjiang, or Tibet who have never received JE vaccination are recommended to receive 1 dose of JE-L prior to travelling to other provincial-level administrative divisions (PLADs) during the JE epidemic season, or prior to moving to settle down in another PLAD.

There should be a 3-month interval between receiving immunoglobulin and being vaccinated with JE-L vaccine.

\section{Catch-up vaccination}

Age-eligible children born after 2007, when JE-L vaccine was included into EPI, who have not received JE-L should be given 2 doses of JE-L (if JE-L vaccination is chosen) with a minimum interval of 12 months between doses.

\section{Schedule}

\section{JE-I Vaccine}

JE-I is administered in a 4-dose series, with 2 doses give at 8 months with an interval of 7 to 10 days, 1 dose given at 2 years, and 1 dose given at 6 years. JE-I is given by $\mathrm{IM}$ in a dose amount of $0.5 \mathrm{~mL}$.

\section{Notes}

There is a 1-month minimum interval between 
receipt of immunoglobulin and vaccination with JE-I.

3. Catch-up vaccination

Age-eligible children who were born after JE-I vaccine was included into EPI (2007) and have not received JE-I vaccine should be given 4 doses of JE-I (if JE-I vaccine is chosen) with a 7-to-10-day interval between the first dose and the second dose, a 1-12month interval between the second dose and the third dose, and minimum 3-year interval between the third dose and the fourth dose.

\section{MPSV-A and MPSV-AC Vaccines}

\section{Schedule}

MPSV-A and MPSV-AC vaccines are given in a 4dose series: 2 doses of MPSV-A (a dose at 6 months and another dose at 9 months) followed by 2 doses of MPSV-AC (a dose at 3 years and another dose at 6 years). MPSV-A and MPSV-AC are given by subcutaneous injection with dose amounts of $0.5 \mathrm{~mL}$.

\section{Notes}

A minimum interval of 3 months is recommended between any 2 doses of MPSV-A. A minimum interval of 12 months is recommended between the second dose of MPSV-A and the first dose of MPSV-AC.

An interval of at least 3 years is recommended between any 2 doses of MPSV-AC. Repeat vaccination should be avoided for 3 years.

If emergency vaccination against meningitis is necessary, an appropriate meningococcal vaccine should be chosen based on epidemiological characteristics and bacteria serogroup caused the epidemic.

Children less than 24 months old are considered to have completed the recommended schedule of MPSVA vaccine if they received valid doses of meningococcal polysaccharide conjugate vaccine (MPCV-AC) according to the vaccine package insert.

Children are considered to have completed the recommended schedule of MPSV-AC vaccine if have received meningococcal vaccines containing serogroups $A$ and $C$ by 3 years and 6 years.

\section{Catch-up vaccination}

Children who were born after MPSV vaccine was included into EPI (2007) and have not received MPSV or completed the prescribed vaccine series should be given an appropriate type of MPSV based on their age. Children less than 24 months old should be given the missed doses of MPSV-A. Children 24 months or above should be given 2 doses of MPSV-AC, and MPSV-A is not necessary. Children 24 months or above who have not received MPSV-A should be given MPSV-AC as soon as possible before 3 years of age; if the child received 1 dose of MPSV-A, he or she should be given MPSV-AC as soon as possible, after a minimum interval of 3 months. Intervals for catch-up doses should comply with the requirements under "notes" above.

\section{Schedule}

\section{HepA-L Vaccine}

HepA-L is given at 18 months of age via SubQ (subcutaneous) injection with a dose amount of either $0.5 \mathrm{~mL}$ or $1.0 \mathrm{~mL}$, depending on the vaccine package insert.

\section{Notes}

Children who have received 2 or more doses of HepA-I-containing vaccine are considered to have completed the HepA vaccine series and do not need HepA-L vaccine.

There is a 3-month minimum interval between administration of immunoglobulin and vaccination with HepA-L vaccine.

\section{Catch-up vaccination}

Age-eligible children should be given 1 dose of HepA-L (if HepA-L vaccine is chosen) if they were born after HepA vaccine was included into EPI (2007) and have not received HepA.

\section{Schedule}

\section{HepA-I Vaccine}

HepA-I is given as a 2-dose series: the first dose at 18 months and the second dose at 24 months. HepA-I is given by IM (intramuscular) injection in a dose amount of $0.5 \mathrm{~mL}$.

\section{Notes}

Children should be considered as to have completed the HepA-I vaccination series if they have received 2 or more doses of a HepA-I-containing combination vaccine.

\section{Catch-up vaccination}

Age-eligible children born after HepA vaccine was included into EPI (2007) and have not received HepA should be given 2 doses of HepA-I (if HepA-I vaccine is chosen) with a minimum interval of 6 months between doses.

If the second dose of HepA-I vaccine is not available, children who received a single dose of HepAI vaccine may complete their HepA vaccination series by administration of 1 dose of HepA-L after a minimum interval of 6 months following HepA-I 
administration.

\section{VACCINATIONS FOR CHILDREN WITH MEDICAL CONDITIONS}

\section{Prematurely Born and Low Birthweight Infants}

Preterm (delivery before 37 weeks of gestation) and/or low birthweight (less than 2,500 grams at birth) infants should be vaccinated at their calendar age if they are assessed to be medically stable and are continuously improving medically (i.e., without requiring ongoing treatment of severe infection, metabolic diseases, acute kidney disease, liver disease, cardiovascular disease, neurological, or respiratory diseases). For BCG vaccination, see BCG vaccine notes, above.

\section{Allergy}

An "allergic constitution" is not a contraindication to vaccination. Children who have a severe allergy to any vaccine ingredient or have previously had throat swelling, anaphylactic shock, or any other severe systemic allergic reaction to a vaccine component are not permitted to receive the same type of vaccine.

\section{Children Born to Mothers Living with HIV}

For purposes of immunization, children are in 1 of 3 human immunodeficiency virus (HIV) infection statuses after being born to an HIV-infected mother: (1) HIV-infected children; (2) children with unknown HIV status; and (3) non-HIV-infected children. Medical institutions make a diagnosis of whether a child is HIV-infected or has any symptom or sign of immunosuppression. Children younger than 18 months who were born to an HIV-infected mother do not need to be screened for HIV prior to vaccination, but should be managed as having unknown HIV status, as described in the table below, until their HIV status is determined.

Children born to an HIV-infected mothers should not be vaccinated with BCG vaccine immediately after birth. BCG should be offered only if the infant is confirmed to be not HIV-infected.

Children born to HIV-infected mothers who have been diagnosed with AIDS-related symptoms or who have symptoms or signs of immune deficiency according to medical institutions should not be vaccinated with any measles-containing vaccine; measles-containing vaccines can be given to children without AIDS-related symptoms.

Children born to HIV-infected mothers can be

TABLE 2. Recommended national immunization program vaccines for children born to HIV-infected mothers.

\begin{tabular}{|c|c|c|c|c|c|}
\hline \multirow[b]{2}{*}{ Vaccine } & \multicolumn{2}{|c|}{ HIV-infected children } & \multicolumn{2}{|c|}{ Children with unknown HIV status } & \multirow{2}{*}{$\begin{array}{l}\text { Non-HIV-infected } \\
\text { children }\end{array}$} \\
\hline & $\begin{array}{c}\text { With symptoms or } \\
\text { immunosuppression }\end{array}$ & $\begin{array}{l}\text { Without symptoms or } \\
\text { immunosuppression }\end{array}$ & $\begin{array}{c}\text { With symptoms or } \\
\text { immunosuppression }\end{array}$ & $\begin{array}{c}\text { Without } \\
\text { symptoms }\end{array}$ & \\
\hline НерВ & $\sqrt{ }$ & $\sqrt{ }$ & $\sqrt{ }$ & $\sqrt{ }$ & $\sqrt{ }$ \\
\hline BCG & $x$ & $x$ & Delay vaccination & $\begin{array}{c}\text { Delay } \\
\text { vaccination }\end{array}$ & $\sqrt{ }$ \\
\hline IPV & $\sqrt{ }$ & $\sqrt{ }$ & $\sqrt{ }$ & $\sqrt{ }$ & $\sqrt{ }$ \\
\hline bOPV & $x$ & $x$ & $x$ & $x$ & $\sqrt{ }$ \\
\hline DTaP & $\sqrt{ }$ & $\sqrt{ }$ & $\sqrt{ }$ & $\sqrt{ }$ & $\sqrt{ }$ \\
\hline DT & $\sqrt{ }$ & $\sqrt{ }$ & $\sqrt{ }$ & $\sqrt{ }$ & $\sqrt{ }$ \\
\hline MMR & $x$ & $\sqrt{ }$ & $x$ & $\sqrt{ }$ & $\sqrt{ }$ \\
\hline JE-I & $\sqrt{ }$ & $\sqrt{ }$ & $\sqrt{ }$ & $\sqrt{ }$ & $\sqrt{ }$ \\
\hline JE-L & $x$ & $x$ & $\times$ & $x$ & $\sqrt{ }$ \\
\hline MPSV-A & $\sqrt{ }$ & $\sqrt{ }$ & $\sqrt{ }$ & $\sqrt{ }$ & $\sqrt{ }$ \\
\hline MPSV-AC & $\sqrt{ }$ & $\sqrt{ }$ & $\sqrt{ }$ & $\sqrt{ }$ & $\sqrt{ }$ \\
\hline HepA-L & $x$ & $x$ & $x$ & $x$ & $\sqrt{ }$ \\
\hline HepA-I & $\sqrt{ }$ & $\sqrt{ }$ & $\sqrt{ }$ & $\sqrt{ }$ & $\sqrt{ }$ \\
\hline
\end{tabular}

Note: Delay vaccination: Supplemental vaccination should be offered to children if HIV antibody has been confirmed negative, and should not be given to children whose HIV antibody is confirmed positive. "ل" means "no special contraindications", and "x" means "no vaccination." $\mathrm{HepB}=$ Hepatitis B vaccine; $\mathrm{BCG}=$ Bacillus Calmette-Guerin vaccine; IPV=Inactivated Polio vaccine; bOPV=Bivalent Oral Polio vaccine; $\mathrm{DTaP}=$ Diphtheria, tetanus, and pertussis combination vaccine; DT=Diphtheria-Tetanus vaccine; MMR=Measles, mumps, and rubella vaccine; JE-I=Japanese encephalitis vaccine, inactivated; JE-L=Japanese encephalitis vaccine, live; MPSV-A=Group A meningococcal polysaccharide vaccine; MPSV-AC=Group $A$ and Group $C$ meningococcal polysaccharide vaccine; HepA-L=Hepatitis $A$ vaccine, live; HepAI=Hepatitis A vaccine, inactivated. 
vaccinated with HepB, DTaP, MPSV-A, MPSV-AC, and DT according to the immunization schedule.

Children born to HIV-infected mothers should not be vaccinated with JE-L, HepA-L, or bOPV unless they are clearly not HIV infected. The inactivated versions of these three vaccines JE-I, HepA-I, and IPV can be given according to the immunization schedule.

Children born to non-HIV-infected mothers do not need routine HIV screening before vaccination. If a child has exposure risk and is diagnosed with HIV infection, follow-up vaccination should follow the vaccination recommendations for HIV-infected children in the attached table.

Please refer to the table below for recommendations of NIP vaccines for children according to HIV status.

\section{Immune Function Abnormalities}

Children with immunodeficiencies or who are undergoing systemic immunosuppressive therapy, excluding HIV-infected children as per above, as a principle, should receive inactivated vaccines rather than live vaccines. This principle does not apply to patients with complement deficiency.

\section{Other Medical Conditions}

The following conditions are not contraindications to vaccination: physiological jaundice, breast-milk jaundice, history of simple febrile convulsions, epilepsy controlled by medication, static neurologic disorders, liver disease, common congenital diseases (e.g., congenital hypothyroidism, phenylketonuria, Down syndrome, congenital heart disease), and congenital infections (e.g., syphilis, cytomegalovirus, and rubella).

Children with other medical conditions can be vaccinated according to the immunization schedule, in principle, if there is no clear evidence that vaccination poses safety risks.

Acknowlegements: The National Immunization Program Technical Working Group members for their contributions developing and writing document; Dr. Lance Rodewald for English polishing.

doi: $10.46234 / \mathrm{ccdcw} 2021.270$

Submitted: December 16, 2021; Accepted: December 20, 2021 